\title{
Efficacy and safety of saxagliptin in patients with type 2 diabetes: A systematic review and meta-analysis
}

\author{
Peng Men, Xiao-tong Li, Hui-lin Tang, Suo-di Zhai* \\ Department of Pharmacy, Peking University Third Hospital, Beijing, China \\ *zhaisuodi@163.com
}

\section{Abstract}

\section{Objective}

To evaluate the comparative efficacy and safety of saxagliptin for type 2 diabetes (T2D).

\section{Methods}

A systematic search of PubMed, Embase, the Cochrane Library, Web of Science, ClinicalTrials.gov and two Chinese databases for randomized controlled trials (RCTs) comparing saxagliptin with placebo or active comparators was performed up to July 2017. A complementary search was done to cover literature until March 2018. For continuous data, estimates were pooled using inverse variance methodology to calculate weighted mean differences (WMDs). Dichotomous data were presented as Mantel-Haenzel risk ratios (RRs).

\section{Results}

Thirty-nine references of 30 RCTs involving 29,938 patients were analyzed. Compared with placebo, saxagliptin significantly reduced glycated hemoglobin ( $\mathrm{HbA1c}, \mathrm{WMD}$ $-0.52 \%, 95 \% \mathrm{Cl}-0.60$ to -0.44 ) and fasting plasma glucose (WMD $-13.78 \mathrm{mg} / \mathrm{dL}, 95 \% \mathrm{Cl}$ -15.31 to -12.25 ), and increased the proportion of patients achieving $\mathrm{HbA} 1 \mathrm{c}<7 \%$ (RR $1.64,95 \% \mathrm{Cl} 1.53$ to 1.75 ). When combined with submaximal-dose metformin, saxagliptin significantly increased the proportion of patients achieving $\mathrm{HbA} 1 \mathrm{c}<7 \%$ compared with acarbose (RR 2.38, 95\% Cl 1.17 to 4.83) and uptitrated metformin (RR 1.30, 95\% Cl 1.04 to 1.63). Saxagliptin was similar to other DPP-4 inhibitors but inferior to liraglutide and dapagliflozin on glycemic control. Saxagliptin significantly decreased the incidences of overall adverse events compared with acarbose (RR $0.71,95 \% \mathrm{Cl} 0.57$ to 0.89 ) and liraglutide (RR $0.41,95 \% \mathrm{Cl} 0.24$ to 0.71 ) when added to metformin. Weight gain and hypoglycemia with saxagliptin was slightly but significantly higher than placebo and lower than sulfonylureas. Saxagliptin did not increase the risk of arthralgia, heart failure, pancreatitis and other adverse events.

Competing interests: The authors have declared that no competing interests exist. 


\section{Conclusions}

Generally, saxagliptin has similar efficacy compared with most oral antidiabetic drugs and may be more effective than acarbose, while having a better safety profile than both acarbose and sulfonylureas.

\section{Introduction}

Type 2 diabetes (T2D) is a chronic disease rapidly increasing in prevalence that imposing enormous medical and economic burdens on on individuals, families, and national health systems worldwide. It is estimated that approximately 415 million people in the world had diabetes in 2015, and this figure is projected to increase to 642 million by 2040 [1]. Health spending on diabetes accounted for $11.6 \%$ of total health expenditure worldwide in 2015 [2]. A diversity of antidiabetic drugs to treat the condition is now available, including metformin, sulfonylureas, thiazolidinediones, $\alpha$-glucosidase inhibitors, prandial glucose regulators, sodiumglucose cotransporter 2 (SGLT2) inhibitors, and insulin in various forms. Because of the progressive nature of diabetes, clinicians and patients often experience difficulty in achieving and sustaining glycemic control. Utilization of antidiabetic drugs should be based on the individual patient's characteristics and preferences and balance the need to optimize the benifits of glycaemic control with the need to limit the risk of adverse effects.

Dipeptidyl peptidase 4 (DPP-4) inhibitors (gliptins) are a relatively new class of oral antidiabetic drugs for the treatment of type 2 diabetes. They act by increasing postprandial concentrations of glucagon-like peptide-1 (GLP-1) and glucose-dependent insulinotropic peptide (GIP) [3-4]. GLP-1 and GIP stimulate insulin secretion in a glucose-dependent manner, suppressing glucagon secretion and slowing gastric emptying. The American Diabetes Association and the European Association for the Study of Diabetes have advocated the use of DPP-4 inhibitors as first-line agent in circumstances where metformin is contraindicated or not tolerated, or within a dual or triple agent regimen [5]. Saxagliptin is an orally active, once-daily, selective and reversible inhibitor of DPP-4 enzyme indicated/approved as an adjunct to diet and exercise to improve glycemic control in adults with type 2 diabetes [6,7].

With the growing number of pharmacological options for treating type 2 diabetes, there is a need for state-of-the-art evidence to inform clinical decisions about differences among the various medications. Recent systematic reviews and meta analyses of DPP-4 inhibitors have mainly focused on the clinical profiles of these drugs as a whole class [8-11]. For saxagliptin, only one meta-analysis [12] examined its efficacy based on 14 phase 2 and 3 trials, without systematically database searches. This systematic review synthesized currently available evidence to provide a better understanding of the comparative efficacy and safety of saxagliptin in treating type 2 diabetes.

\section{Methods}

\section{Data sources and search strategy}

This meta-analysis of the available data on saxagliptin was undertaken using a predetermined protocol, and is reported in accordance with the Preferred Reporting Items for Systematic Reviews and Meta-Analyses (PRISMA) statement (see S1 Text) [13]. Relevant studies for the analysis were selected by searching PubMed, Embase, the Cochrane Library, Web of Science, and 2 Chinese databases [China National Knowledge Infrastructure (CNKI) and Chinese Biomedical Literature Database (CBM)] up to the end of July 2017. A complementary search was 
performed in order to include the most recent articles (published before March 2018). The generic drug name "saxagliptin" was used as the only search term. If necessary, specific filters for retrieving randomized controlled trials (RCTs) conducted in humans were incorporated into the search string. We considered all potentially eligible studies for review, irrespective of the primary outcomes or language. We also performed a manual search using the reference lists of published reviews of saxagliptin.

The research questions and eligibility criteria for the systematic review conformed with the PICOS (participants, interventions, comparators, outcomes and study design) approach. Studies meeting the following criteria were considered for inclusion:

- Participants: patients over 18 years of age with type 2 diabetes.

- Interventions: saxagliptin used in the treatment of type 2 diabetes (as monotherapy or in dual or triple therapy).

- Comparators: placebo or other active antidiabetic interventions (as monotherapy or in dual or triple therapy).

- Outcomes: glycated hemoglobin (HbAlc), proportion of patients achieving $\mathrm{HbAlc}$ targets of $<7 \%$, fasting plasma glucose (FPG) concentration, overall and serious adverse events, body weight, confirmed hypoglycemia, heart failure, pancreatitis, arthralgia, and other adverse events [hypertension, urinary tract infection, upper respiratory tract infection, nasopharyngitis]. For continuous data, the mean change from baseline was examined.

- Study type: RCTs involving $>150$ patients.

\section{Study selection and data extraction}

The titles and abstracts of all retrieved citations were independently screened by 2 reviewers to identify potentially relevant studies. The full texts of relevant citations were then retrieved to determine their suitability for inclusion. If there were any discrepancies between the 2 reviewers, a third reviewer became involved.

Data were independently abstracted by the 2 principal reviewers and any discrepancies were resolved by consensus. The data extracted included study characteristics (design, total number of participants, trial duration, antidiabetic treatments, patient inclusion and exclusion criteria), patient demographics (age, sex, baseline HbAlc levels), and pre-specified efficacy and safety outcomes. For extension studies, if the treatment assignment was switched from placebo to saxagliptin, only the outcome data up to that point were documented. Only information that related to dosages currently approved by the US Food and Drug Administration (FDA) or the European Medicines Agency (EMA) were abstracted. Corresponding authors were contacted for data not provided within studies, or when outcomes were presented in an unsuitable format for data synthesis.

\section{Quality assessment}

Two reviewers independently applied the Cochrane Risk of Bias tool to assessed the risk of bias in the RCTs. The following methodological features relevant to the minimization of bias were assessed: randomization, random allocation concealment, masking of treatment allocation, blinding, incomplete outcomes data, selective reporting, and other items. The following judgements were used: low risk, high risk, or unclear risk (either lack of information or uncertainty regarding the potential for bias). Disagreements were resolved by consensus with a third reviewer. 


\section{Data synthesis and analysis}

Outcomes were pooled using Review Manager 5.2 software (RevMan, Cochrane, London, UK). For continuous data, estimates were pooled using inverse variance methodology to calculate weighted mean differences (WMDs). Dichotomous data were presented as Mantel-Haenzel risk ratios (RRs). All results were estimated from each study with $95 \%$ confidence intervals (CIs). Heterogeneity was assessed using the chi-square test and the $\mathrm{I}^{2}$ statistic. If $\mathrm{I}^{2}$ was $50 \%$, a fixed-effect model with the Mantel-Haenszel method was used; otherwise, the random-effect model was adopted.

Where studies did not report standard deviations (SDs) explicitly, these were derived from the available published information. If possible, standard errors (SEs) were calculated from CIs. If these data were unavailable, they were derived from $p$-values for changes from baseline. All trials were included in at least one of the analyses, and each trial could be used in multiple sets of analyses. Data were reported first from studies involving placebo comparisons, and then from studies involving active comparators.

\section{Results}

The selection process for articles included in the systematic review is shown in Fig 1. From the 5773 citations identified by the initial and complementary literature searching, 38 references [14-51], including 30 trials involving 29,938 participants, ultimately met the inclusion criteria for the meta-analysis. The study characteristics and the demographics of the patient populations in the retrieved studies were comparable (Table 1). Enrolled patients in these RCTs had a mean age of 42.0 to 72.6 years. The mean duration of type 2 diabetes ranged from 0.4 to 16.7 years, with mean baseline $\mathrm{HbA1c}$ levels between $7.6 \%$ and $10.7 \%$.

\section{Quality of the included studies}

A risk of bias summary is shown in S1a Fig, and an assessment of the risk of bias for each of the studies selected is shown in S1b Fig. Random sequence generation was adequate in 25 trials, and allocation concealment was adequately described in 16 trials. Two trials were considered to be at high risk of performance and detection bias. All studies were judged to be at low risk of attrition, reporting and other bias.

\section{Glycemic control}

Glycated hemoglobin (HbAlc). In comparison with placebo, saxagliptin produced significantly greater reductions in HbAlc (WMD $-0.52 \%, 95 \%$ CI -0.60 to $-0.44 ; p<0.00001$; Fig $2 \mathrm{a}$ ), whether used as monotherapy (WMD $-0.50 \%, 95 \% \mathrm{CI}-0.62$ to $-0.37 ; p<0.00001$ ) or add-on therapy (WMD $-0.52 \%, 95 \% \mathrm{CI}-0.62$ to $-0.43 ; p<0.00001$ ). Both the $2.5 \mathrm{mg} /$ day and $5 \mathrm{mg} /$ day dosages of saxagliptin produced significant improvements in $\mathrm{HbAlc}$, with similar absolute effect sizes.

Overall, saxagliptin produced similar reduction in HbAlc compared with active comparators when added to metformin (WMD $0.01 \%, 95 \%$ CI -0.11 to 0.13 ; $p=0.89$; Fig $2 \mathrm{~b}$ ). Saxagliptin significantly reduced $\mathrm{HbA1c}$ in comparison with acarbose (WMD $-0.12 \%, 95 \% \mathrm{CI}-0.22$ to $-0.02 ; p=0.02$ ), but not compared with liraglutide (WMD $0.27 \%$, 95\% CI 0.09 to 0.45 ; $p=0.003$ ) or dapagliflozin (WMD $0.32 \%, 95 \%$ CI 0.11 to $0.53 ; p=0.003$ ). Saxagliptin produced similar reduction compared with metformin (WMD - $0.30 \%, 95 \%$ CI -0.74 to $0.13 ; p=0.17$ ), sulfonylureas (WMD $0.14 \%$, 95\% CI -0.02 to $0.30 ; p=0.08$ ), sitagliptin (WMD $0.10 \%$, 95\% CI -0.01 to $0.20 ; p=0.07$ ), vildagliptin (WMD $0.02 \%, 95 \%$ CI -0.15 to $0.19 ; p=0.79$ ) and repaglinide (WMD $0.30 \%, 95 \%$ CI -0.18 to $0.78 ; p=0.22$ ). 
5199 articles identified through database searching (PubMed:541, the Cochrane Library:220, EMbase:2305, Web of Science:1120, ClinicalTrials.gov: 127, CNKI: 392, WanFang: 494)
574 articles identified through complementary searching (PubMed:55, the Cochrane Library:32, EMbase:193, Web of Science:110, ClinicalTrials.gov:

1, CNKI: 100, WanFang: 83)

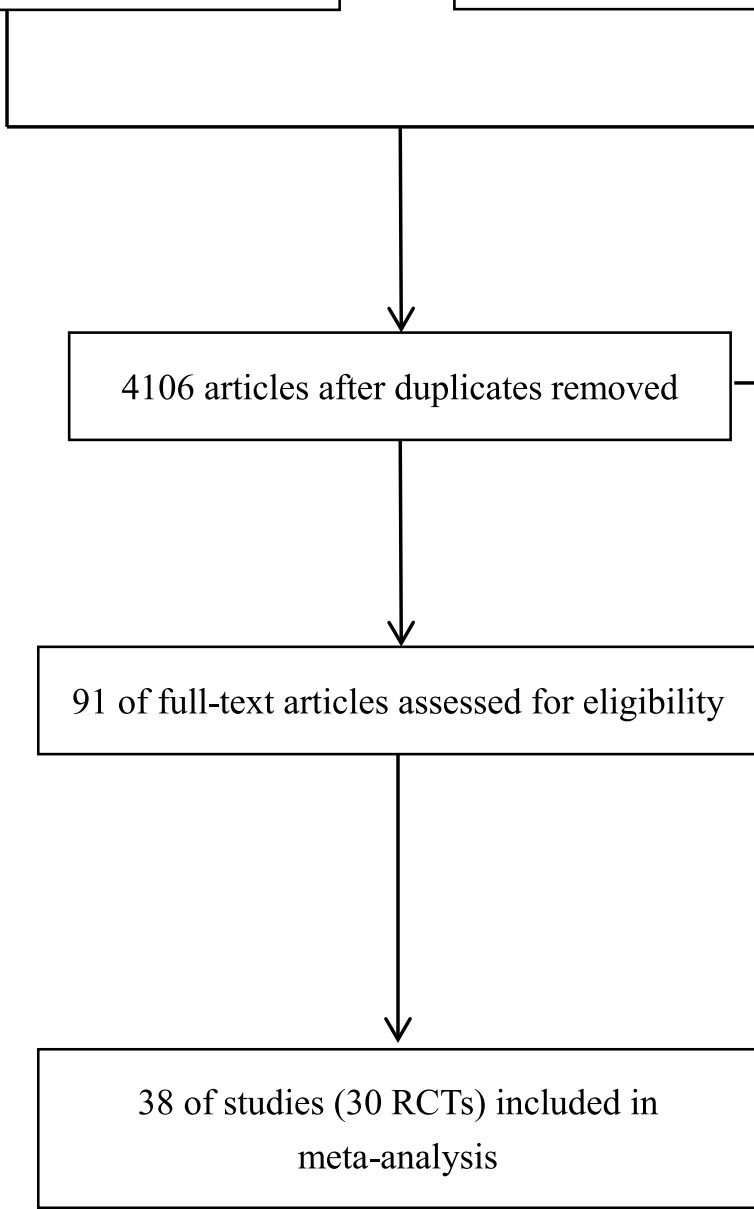

1323 articles excluded

48 articles without enough enrolled patients

2 articles without full text

3 articles without comparators

Fig 1. Selection process for articles in the systematic review.

https://doi.org/10.1371/journal.pone.0197321.g001

A significantly greater proportion of patients treated with saxagliptin also achieved a target HbA1c of $<7 \%$ compared with placebo (RR 1.64, 95\% CI 1.53 to 1.75 ; $p<0.00001$; S2a Fig), whether used as monotherapy (RR $1.53,95 \%$ CI 1.32 to $1.77 ; \mathrm{p}<0.00001$ ) or add-on therapy (RR 1.67, 95\% CI 1.55 to $1.81 ; p<0.00001)$. Similar results was shown when compared with metformin (RR 1.30, 95\% CI 1.04 to $1.63 ; p=0.02$ ) and acarbose (RR 2.38, 95\% CI 1.17 to 4.83; $p=0.02)$. However, no significant differences were observed in comparisons of saxagliptin with other active comparators (S2b Fig).

Fasting plasma glucose (FPG). In comparison with placebo, saxagliptin produced significantly greater reductions in FPG (WMD $-13.78 \mathrm{mg} / \mathrm{dL}, 95 \% \mathrm{CI}-15.31$ to $-12.25 ; p<0.00001$; Fig 3a), whether used as monotherapy (WMD $-14.89 \mathrm{mg} / \mathrm{dL}, 95 \% \mathrm{CI}-20.14$ to -9.64 ; $p<0.00001$ ) or add-on therapy to other antidiabetic agents (WMD $-13.68 \mathrm{mg} / \mathrm{dL}, 95 \%$ 
Table 1. Patient demographics and study characteristics of the included studies.

\begin{tabular}{|c|c|c|c|c|c|c|c|c|c|c|c|c|}
\hline $\begin{array}{l}\text { Trial } \\
\text { No. }\end{array}$ & Reference, year & $\begin{array}{l}\text { Clinicaltrial. } \\
\text { gov No. }\end{array}$ & $\begin{array}{l}\text { Trial } \\
\text { duration } \\
\text { (weeks) }\end{array}$ & $\mathbf{n}$ & $\begin{array}{l}\text { Mean } \\
\text { age } \\
\text { (years) }\end{array}$ & $\begin{array}{l}\text { Sex } \\
\text { (male/ } \\
\text { female) }\end{array}$ & $\begin{array}{l}\text { Baseline } \\
\text { HbAlc } \\
(\%)\end{array}$ & $\begin{array}{l}\text { Baseline } \\
\text { FPG }(\mathrm{mg} / \\
\text { dL) }\end{array}$ & $\begin{array}{l}\text { Baseline } \\
\text { body } \\
\text { weight } \\
(\mathrm{kg})\end{array}$ & $\begin{array}{l}\text { T2D } \\
\text { duration } \\
\text { (years) }\end{array}$ & Interventions & $\begin{array}{l}\text { Added-on } \\
\text { to }\end{array}$ \\
\hline \multirow[t]{2}{*}{1} & $\begin{array}{l}\text { Barnett et al., } \\
2012 \text { [14] }\end{array}$ & NCT00757588 & 24 & \multirow[t]{2}{*}{455} & \multirow[t]{2}{*}{57.3} & \multirow[t]{2}{*}{$188 / 267$} & \multirow[t]{2}{*}{8.7} & \multirow[t]{2}{*}{173.4} & \multirow[t]{2}{*}{87.2} & \multirow[t]{2}{*}{11.9} & \multirow[t]{2}{*}{ SAXA 5 mg/d PLB } & \multirow[t]{2}{*}{ INS } \\
\hline & $\begin{array}{l}\text { Barnett et al., } \\
2013 \text { [15] }\end{array}$ & & 52 & & & & & & & & & \\
\hline \multirow[t]{2}{*}{2} & $\begin{array}{l}\text { Chacra et al., } \\
2009 \text { [16] }\end{array}$ & NCT00313313 & 24 & \multirow[t]{2}{*}{768} & \multirow[t]{2}{*}{55.0} & \multirow[t]{2}{*}{$346 / 422$} & \multirow[t]{2}{*}{8.4} & \multirow[t]{2}{*}{173.4} & \multirow[t]{2}{*}{75.7} & \multirow[t]{2}{*}{6.9} & \multirow[t]{2}{*}{$\begin{array}{l}\text { SAXA } 2.5 \mathrm{mg} / \mathrm{d} \\
\text { SAXA } 5 \mathrm{mg} / \mathrm{d} \text { PLB }\end{array}$} & \multirow[t]{2}{*}{ GLY } \\
\hline & $\begin{array}{l}\text { Chacra et al., } \\
2011 \text { [1]] }\end{array}$ & & 76 & & & & & & & & & \\
\hline 3 & $\begin{array}{l}\text { Chen et al., } \\
2017 \text { [18] }\end{array}$ & NCT02104804 & 24 & 462 & 59.1 & $209 / 253$ & 8.5 & 167.6 & NA & 13.4 & SAXA 5 mg/d PLB & INS \pm MET \\
\hline \multirow[t]{2}{*}{4} & $\begin{array}{l}\text { DeFronzo } \\
\text { et al., 2009 [19] }\end{array}$ & NCT00121667 & 24 & \multirow[t]{2}{*}{743} & \multirow[t]{2}{*}{54.6} & \multirow[t]{2}{*}{ NA } & \multirow[t]{2}{*}{8.0} & \multirow[t]{2}{*}{176.0} & \multirow[t]{2}{*}{ NA } & \multirow[t]{2}{*}{6.5} & \multirow{2}{*}{$\begin{array}{l}\text { SAXA } 2.5 \mathrm{mg} / \mathrm{d} \\
\text { SAXA } 5 \mathrm{mg} / \mathrm{d} \\
\text { SAXA } 10 \mathrm{mg} / \mathrm{d} \text { PLB }\end{array}$} & \multirow[t]{2}{*}{ MET } \\
\hline & $\begin{array}{l}\text { Rosenstock } \\
\text { et al., } 2013[20]\end{array}$ & & 206 & & & & & & & & & \\
\hline 5 & $\begin{array}{l}\text { Dou et al., } \\
2017 \text { [21] }\end{array}$ & NCT02273050 & 24 & 630 & 50.1 & $419 / 211$ & 9.4 & 182.7 & 73.8 & 0.81 & SAXA $5 \mathrm{mg} / \mathrm{d}$ PLB & MET \\
\hline 6 & $\begin{array}{l}\text { Du et al., } 2017 \\
\text { [22] }\end{array}$ & NCT02243176 & 24 & 481 & 55.6 & $285 / 196$ & 8.2 & 160.3 & 72.9 & 5.2 & $\begin{array}{l}\text { SAXA } 5 \mathrm{mg} / \\
\text { dACBO } 300 \mathrm{mg} / \mathrm{d}\end{array}$ & MET \\
\hline 7 & $\begin{array}{l}\text { Fonseca et al., } \\
2012[23]\end{array}$ & NCT00960076 & 18 & 282 & 55.3 & $130 / 152$ & 8.4 & 162.9 & NA & 6.2 & $\begin{array}{l}\text { SAXA } 5 \mathrm{mg} / \mathrm{d} \text { MET } \\
\text { (uptitrated to } 2000 \\
\mathrm{mg} / \mathrm{d} \text { ) }\end{array}$ & MET \\
\hline 8 & $\begin{array}{l}\text { Frederich } \\
\text { et al., 2012 [24] }\end{array}$ & NCT00316082 & 76 & 365 & 54.8 & $168 / 197$ & 7.9 & 162.1 & 84.9 & 1.7 & $\begin{array}{l}\text { SAXA } 2.5 \mathrm{mg} / \mathrm{d} \\
\text { SAXA } 5 \mathrm{mg} / \mathrm{d} \text { PLB }\end{array}$ & - \\
\hline \multirow[t]{2}{*}{9} & $\begin{array}{l}\text { Goke et al., } \\
2010[25]\end{array}$ & NCT00575588 & 52 & 858 & 57.6 & $444 / 414$ & 7.7 & 162.0 & 88.7 & 5.5 & $\begin{array}{l}\text { SAXA } 5 \mathrm{mg} / \mathrm{d} \\
\text { GLPZ } 5-20 \mathrm{mg} / \mathrm{d}\end{array}$ & MET \\
\hline & $\begin{array}{l}\text { Goke et al., } \\
2013 \text { [26] }\end{array}$ & & 104 & & & & & & & & & \\
\hline 10 & $\begin{array}{l}\text { Hermans et al., } \\
2012[27]\end{array}$ & NCT01006590 & 24 & 286 & 58.7 & $164 / 122$ & 7.8 & 169.8 & NA & 6.5 & $\begin{array}{l}\text { SAXA } 5 \mathrm{mg} / \mathrm{d} \text { MET } \\
\text { (uptitrated to } 2000 \\
\mathrm{mg} / \mathrm{d} \text { ) }\end{array}$ & MET \\
\hline 11 & $\begin{array}{l}\text { Hollander } \\
\text { et al., 2009 [28] }\end{array}$ & NCT00295633 & 24 & 565 & 54.0 & $280 / 285$ & 8.3 & 162.9 & 81.1 & 5.2 & $\begin{array}{l}\text { SAXA } 2.5 \mathrm{mg} / \mathrm{d} \\
\text { SAXA } 5 \mathrm{mg} / \mathrm{d} \text { PLB }\end{array}$ & TZD \\
\hline & $\begin{array}{l}\text { Hollander } \\
\text { et al. } 2011 \text { [29] }\end{array}$ & & 76 & & & & & & & & & \\
\hline 12 & $\begin{array}{l}\text { Jadzinsky } \\
\text { et al., } 2009 \text { [30] }\end{array}$ & NCT00327015 & 24 & 1306 & 52.0 & $643 / 663$ & 9.5 & 201.3 & 82.5 & 1.7 & $\begin{array}{l}\text { SAXA } 5 \mathrm{mg} / \mathrm{d} \\
\text { SAXA } 10 \mathrm{mg} / \mathrm{d} \text { PLB }\end{array}$ & MET \\
\hline & $\begin{array}{l}\text { Pfutzner et al., } \\
2011 \text { [31] }\end{array}$ & & 76 & & & & & & & & & \\
\hline 13 & $\begin{array}{l}\text { Kadowaki } \\
\text { et al., 2017 [32] }\end{array}$ & 1 & 16 & 240 & 63.4 & $139 / 89$ & 8.3 & 163.1 & 65.5 & 15.8 & SAXA $5 \mathrm{mg} / \mathrm{d}$ PLB & INS \\
\hline 14 & $\begin{array}{l}\text { Kumar et al., } \\
2014 \text { [33] }\end{array}$ & NCT00918879 & 24 & 213 & 49.1 & $120 / 93$ & 8.3 & 151.2 & 69.6 & 0.9 & SAXA $5 \mathrm{mg} / \mathrm{d}$ PLB & - \\
\hline 15 & $\begin{array}{l}\text { Li et al., 2014A } \\
{[34]}\end{array}$ & l & 24 & 207 & 46.6 & $109 / 81$ & 8.7 & 153.1 & 74.4 & NA & $\begin{array}{l}\text { SAXA } 5 \mathrm{mg} / \mathrm{d} \text { SITA } \\
100 \mathrm{mg} / \mathrm{d} \text { VIDA } \\
100 \mathrm{mg} / \mathrm{d}\end{array}$ & $\begin{array}{l}\text { MET } \\
+ \text { another } \\
\text { OAD }\end{array}$ \\
\hline 16 & $\begin{array}{l}\text { Li et al., 2014B } \\
{[35]}\end{array}$ & 1 & 24 & 178 & 47.1 & $109 / 69$ & 8.5 & 160.9 & 75.9 & 5.6 & $\begin{array}{l}\text { SAXA } 5 \mathrm{mg} / \mathrm{d} \\
\text { VIDA } 100 \mathrm{mg} / \mathrm{d} \\
\text { LRAG } 1.2 \mathrm{mg} / \mathrm{d}\end{array}$ & $\mathrm{OAD}$ \\
\hline 17 & $\begin{array}{l}\text { Lv et al., } 2013 \\
{[36]}\end{array}$ & 1 & 12 & 180 & 44.0 & NA & 7.8 & 150.2 & 82.0 & 0.5 & $\begin{array}{l}\text { SAXA } 5 \mathrm{mg} / \mathrm{d} \\
\text { ACBO } 150 \mathrm{mg} / \mathrm{d}\end{array}$ & MET \\
\hline
\end{tabular}


Table 1. (Continued)

\begin{tabular}{|c|c|c|c|c|c|c|c|c|c|c|c|c|}
\hline $\begin{array}{l}\text { Trial } \\
\text { No. }\end{array}$ & Reference, year & $\begin{array}{l}\text { Clinicaltrial. } \\
\text { gov No. }\end{array}$ & $\begin{array}{l}\text { Trial } \\
\text { duration } \\
\text { (weeks) }\end{array}$ & n & $\begin{array}{l}\text { Mean } \\
\text { age } \\
\text { (years) }\end{array}$ & $\begin{array}{l}\text { Sex } \\
\text { (male/ } \\
\text { female) }\end{array}$ & $\begin{array}{l}\text { Baseline } \\
\text { HbAlc } \\
(\%)\end{array}$ & $\begin{array}{l}\text { Baseline } \\
\text { FPG (mg/ } \\
\text { dL) }\end{array}$ & $\begin{array}{l}\text { Baseline } \\
\text { body } \\
\text { weight } \\
(\mathrm{kg})\end{array}$ & $\begin{array}{l}\text { T2D } \\
\text { duration } \\
\text { (years) }\end{array}$ & Interventions & $\begin{array}{l}\text { Added-on } \\
\text { to }\end{array}$ \\
\hline \multirow[t]{2}{*}{18} & $\begin{array}{l}\text { Matthaei et al., } \\
2015 \text { [37] }\end{array}$ & NCT01619059 & 24 & 315 & 54.6 & $149 / 166$ & 7.9 & 161.0 & NA & 7.7 & SAXA 5mg/d PLB & $\begin{array}{l}\text { DAPA } \\
+ \text { MET }\end{array}$ \\
\hline & $\begin{array}{l}\text { Matthaei et al., } \\
2016 \text { [38] }\end{array}$ & & 52 & & & & & & & & & \\
\hline 19 & $\begin{array}{l}\text { Moses et al., } \\
2014 \text { [39] }\end{array}$ & NCT01128153 & 24 & 257 & 57.0 & $154 / 103$ & 8.3 & 159.3 & 81.4 & - & SAXA 5 mg/d PLB & $\mathrm{MET}+\mathrm{SU}$ \\
\hline 20 & $\begin{array}{l}\text { Nowicki et al., } \\
2011[40,41]\end{array}$ & NCT00614939 & 12,52 & 170 & 66.5 & $73 / 97$ & 8.3 & 178.3 & 82.9 & 16.7 & $\begin{array}{l}\text { SAXA } 2.5 \mathrm{mg} / \mathrm{d} \\
\text { PLB }\end{array}$ & - \\
\hline 21 & $\begin{array}{l}\text { Pan et al., } 2012 \\
{[42]}\end{array}$ & NCT00698932 & 24 & 568 & 51.4 & $315 / 253$ & 8.2 & 164.7 & 69.2 & 1.0 & SAXA 5 mg/d PLB & - \\
\hline \multirow[t]{2}{*}{22} & \multirow[t]{2}{*}{$\begin{array}{l}\text { Rosenstock } \\
\text { et al., } 2008 \text { [43] }\end{array}$} & NCT00950599 & 12 & 338 & 54.0 & 197/141 & 7.9 & 164.7 & 89.9 & 1.1 & $\begin{array}{l}\text { SAXA } 2.5 \mathrm{mg} / \mathrm{d} \\
\text { SAXA } 5 \mathrm{mg} / \mathrm{d} \\
\text { SAXA } 10 \mathrm{mg} / \mathrm{d} \\
\text { SAXA } 20 \mathrm{mg} / \mathrm{d} \\
\text { SAXA } 40 \mathrm{mg} / \mathrm{d} \text { PLB }\end{array}$ & - \\
\hline & & & 6 & 85 & 52.1 & $50 / 35$ & 7.7 & 148.7 & 91.2 & 0.4 & $\begin{array}{l}\text { SAXA } 100 \mathrm{mg} / \mathrm{d} \\
\text { PLB }\end{array}$ & - \\
\hline \multirow[t]{2}{*}{23} & $\begin{array}{l}\text { Rosenstock } \\
\text { et al., } 2009 \text { [44] }\end{array}$ & NCT00121641 & \multirow[t]{2}{*}{24} & 401 & 53.5 & 204/197 & 7.9 & 175.0 & 89.8 & 2.6 & $\begin{array}{l}\text { SAXA } 2.5 \mathrm{mg} / \mathrm{d} \\
\text { SAXA } 5 \mathrm{mg} / \mathrm{d} \\
\text { SAXA } 10 \mathrm{mg} / \mathrm{d} \text { PLB }\end{array}$ & - \\
\hline & $\begin{array}{l}\text { Rosenstock } \\
\text { et al., 2013 [20] }\end{array}$ & & & 68 & 49.1 & $79 / 82$ & 10.7 & 241.0 & 91.4 & 3.1 & SAXA $10 \mathrm{mg} / \mathrm{d}$ & - \\
\hline 24 & $\begin{array}{l}\text { Rosenstock } \\
\text { et al., } 2015 \text { [45] }\end{array}$ & NCT01606007 & 24 & 534 & 54.0 & $268 / 266$ & 8.9 & 186 & NA & 7.6 & $\begin{array}{l}\text { SAXA } 5 \mathrm{mg} / \mathrm{d} \\
\text { DAPA } 10 \mathrm{mg} / \mathrm{d} \\
\text { SAXA } 5 \mathrm{mg} / \mathrm{d} \\
+ \text { DAPA } 10 \mathrm{mg} / \mathrm{d}\end{array}$ & MET \\
\hline 25 & $\begin{array}{l}\text { Scheen et al., } \\
2010 \text { [46] }\end{array}$ & NCT00666458 & 18 & 801 & 58.4 & $392 / 409$ & 7.7 & 161.1 & NA & 6.3 & $\begin{array}{l}\text { SAXA } 5 \mathrm{mg} / \mathrm{d} \text { SITA } \\
100 \mathrm{mg} / \mathrm{d}\end{array}$ & MET \\
\hline 26 & $\begin{array}{l}\text { Schernthaner } \\
\text { et al., 2015 [47] }\end{array}$ & NCT01006603 & 52 & 720 & 72.6 & $445 / 275$ & 7.6 & NA & NA & 7.6 & $\begin{array}{l}\text { SAXA } 5 \mathrm{mg} / \mathrm{d} \leq 6 \\
\mathrm{mg} / \mathrm{d}\end{array}$ & MET \\
\hline 27 & $\begin{array}{l}\text { Scirica et al., } \\
2013 \text { [48] }\end{array}$ & NCT01107886 & 109 & 16492 & 65.1 & $\begin{array}{l}11037 / \\
5455\end{array}$ & 8.0 & 156.5 & 87.9 & 10.3 & SAXA 5 mg/d PLB & - \\
\hline 28 & $\begin{array}{l}\text { White et al., } \\
2014 \text { [49] }\end{array}$ & NCT00885378 & 12 & 160 & 55.7 & $85 / 75$ & 8.0 & 163.1 & NA & 6.0 & SAXA 5 mg/d PLB & MET \\
\hline 29 & $\begin{array}{l}\text { Xia et al., } 2014 \\
\text { [50] }\end{array}$ & I & 12 & 240 & 42.0 & $126 / 114$ & 8.3 & 157.5 & NA & 1.0 & $\begin{array}{l}\text { SAXA } 5 \mathrm{mg} / \mathrm{d} \\
\text { REGL } 3 \mathrm{mg} / \mathrm{d}\end{array}$ & MET \\
\hline 30 & $\begin{array}{l}\text { Yang et al., } \\
2011[51]\end{array}$ & NCT00661362 & 24 & 570 & 54.1 & $275 / 295$ & 7.9 & 159.3 & 69.0 & 5.1 & SAXA 5 mg/d PLB & MET \\
\hline
\end{tabular}

ACBO, acarbose; DAPA, dapagliflozin; GLMR, glimepiride; GLPZ, glipizide; GLY, glyburide; INS, insulin; LINA, linagliptin; MET, metformin; NA, not available; PLB, placebo; REGL, repaglinide; SAXA, saxagliptin; SITA, sitagliptin; SU, sulfonylurea; TZD, thiazolidinedione; VIDA, vildagliptin.

CI -15.28 to $-12.08 ; p<0.00001)$. Both the $2.5 \mathrm{mg} /$ day and $5 \mathrm{mg} /$ day dosages of saxagliptin produced significant improvements in FPG.

When added to metformin, saxagliptin produced a significantly smaller reduction in FPG compared with sulfonylureas (WMD $9.05 \mathrm{mg} / \mathrm{dL}, 95 \%$ CI 6.18 to 11.93; $p<0.00001$ ), liraglutide (WMD $7.60 \mathrm{mg} / \mathrm{dL}, 95 \%$ CI 1.76 to $13.44 ; p=0.01$ ) and dapagliflozin (WMD $18.00 \mathrm{mg} /$ dL, 95\% CI 10.10 to $25.90 ; p<0.00001$ ). However, no significant differences were observed when saxagliptin was compared with other active comparators (Fig 3b), including sitagliptin 
(a)

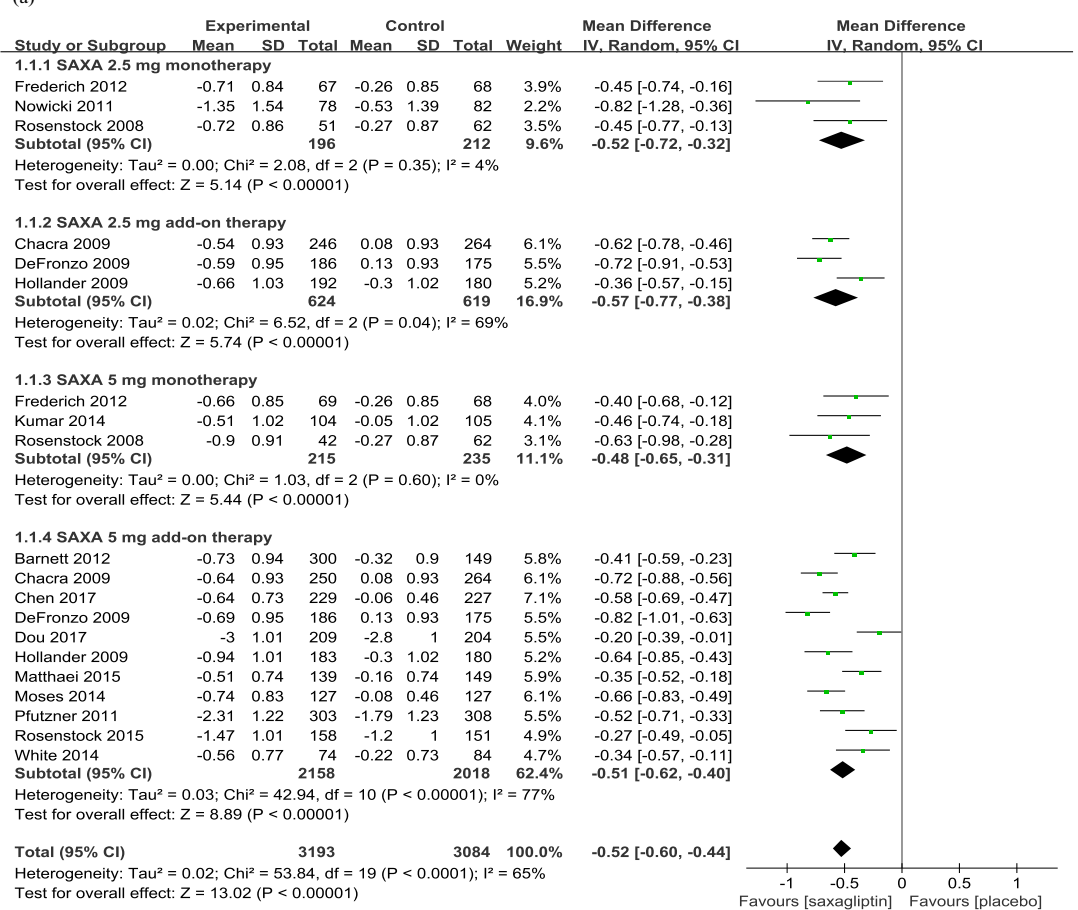

(b)

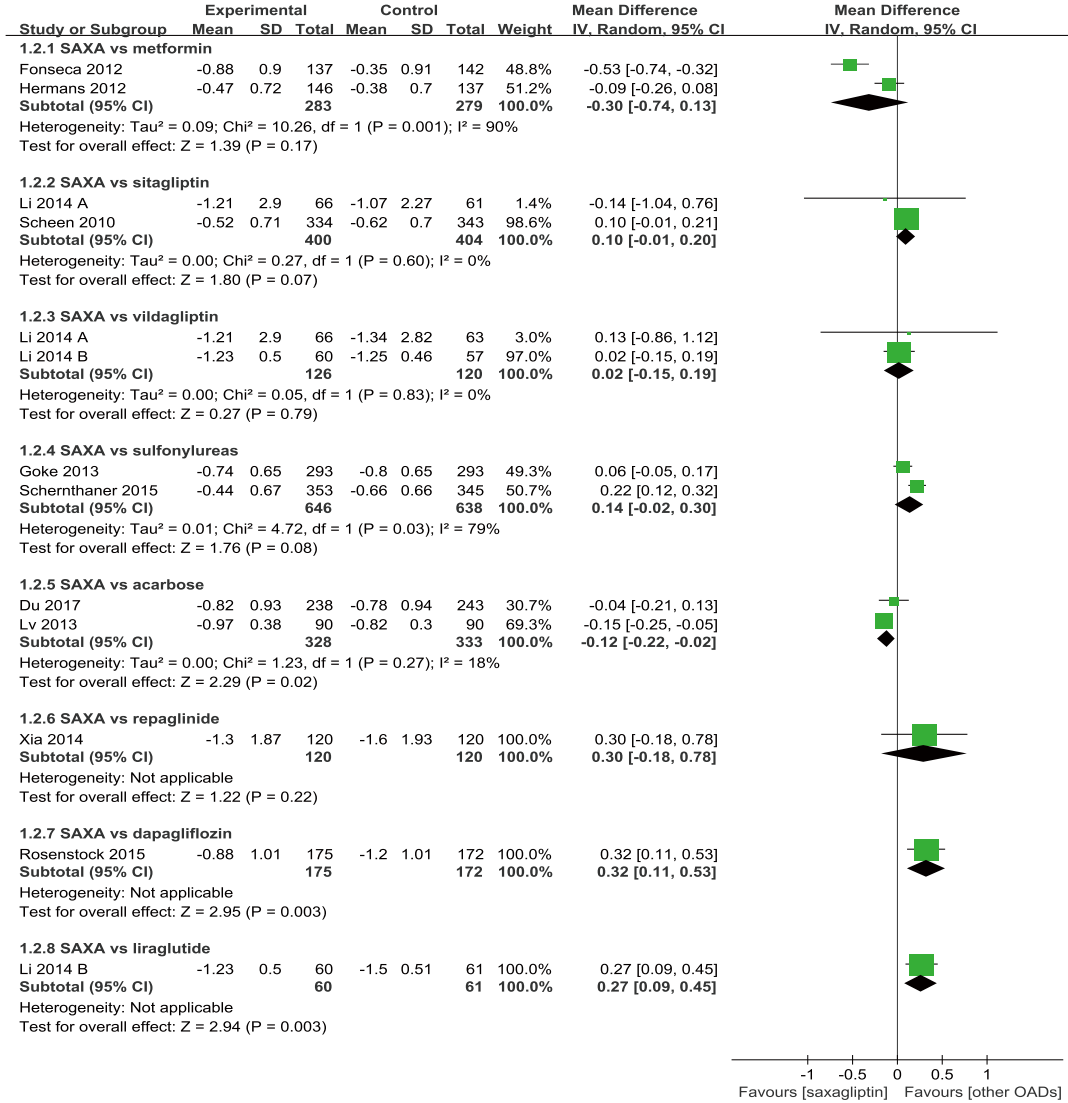

Fig 2. Mean change of HbAlc from baseline (a. saxagliptin vs placebo; b. saxagliptin vs active comparators).

https://doi.org/10.1371/journal.pone.0197321.g002 
(a)

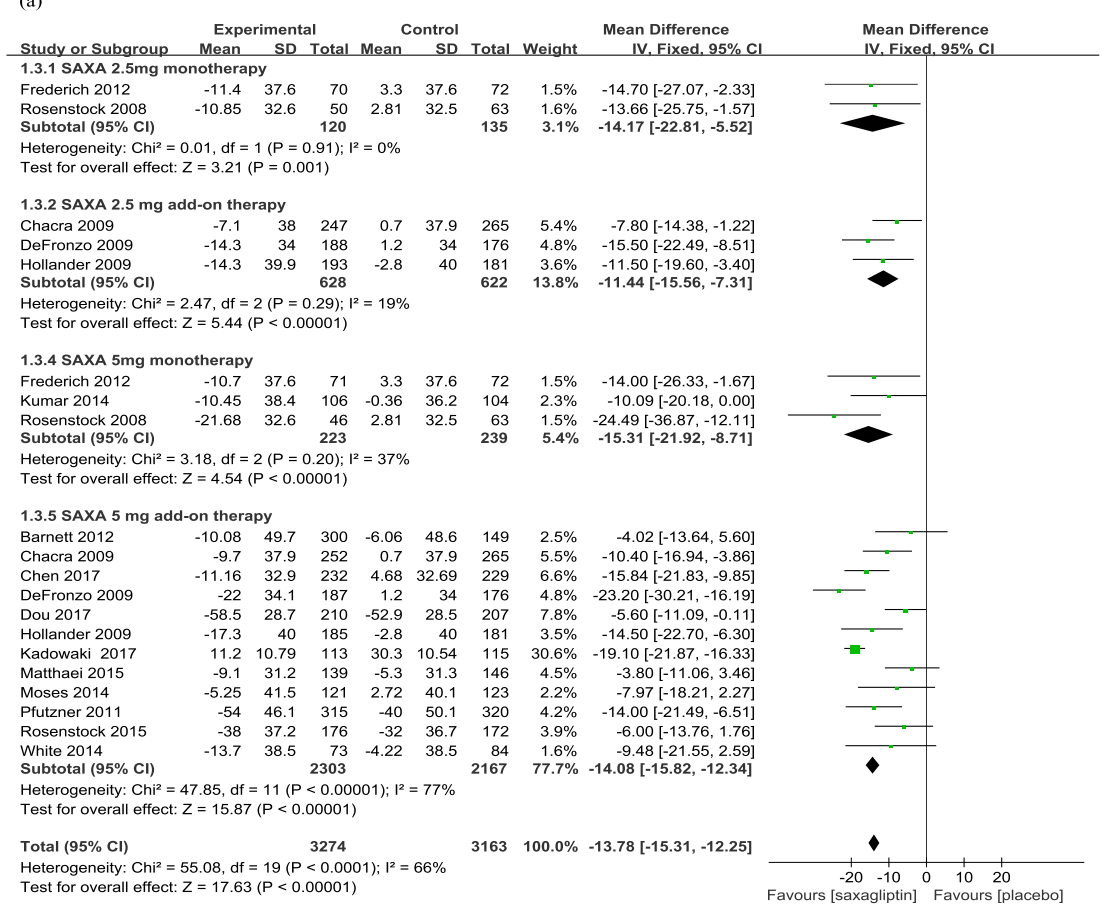

(b)

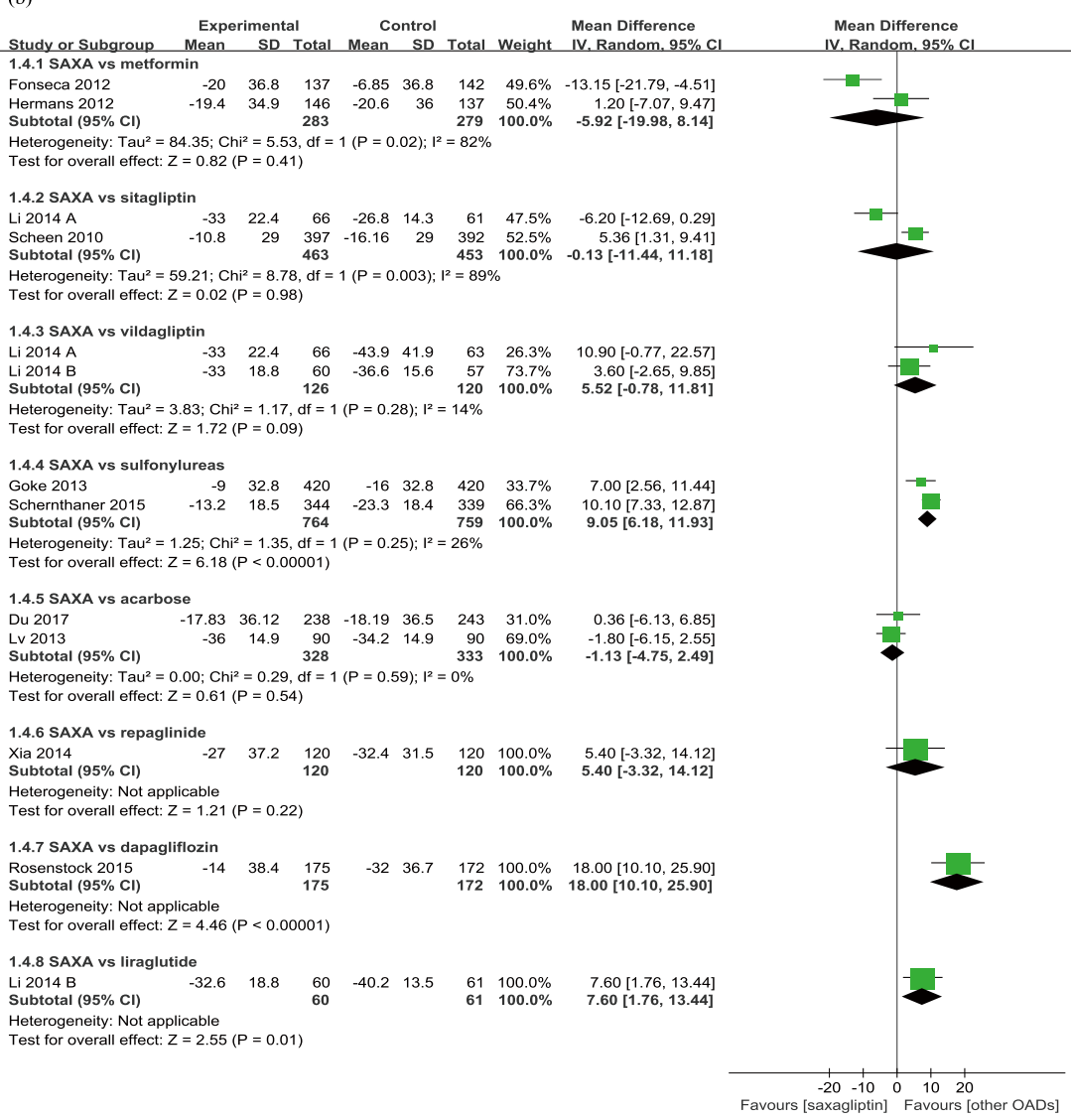

Fig 3. Mean change of FPG from baseline (a. saxagliptin vs placebo; b. saxagliptin vs active comparators).

https://doi.org/10.1371/journal.pone.0197321.g003 
(WMD $-0.13 \mathrm{mg} / \mathrm{dL}, 95 \% \mathrm{CI}-11.44$ to $11.18 ; p=0.98$ ) and vildaglitpin (WMD $5.52 \mathrm{mg} / \mathrm{dL}$, $95 \%$ CI -0.78 to $11.81 ; p=0.09$ ).

\section{Non-glycemic outcomes}

Overall and serious adverse events. Generally, the incidences of overall (versus placobo: RR 1.01, 95\% CI 0.97 to 1.05; $p=0.77$; Fig $4 \mathrm{a}$ ) and severe (versus placobo: RR 1.01, 95\% CI 0.96 to $1.06 ; p=0.78$; S3 Fig) treatment-related adverse events did not increase in the treatment of saxagliptin. Moreover, saxagliptin significantly reduced overall adverse events compared with acarbose (RR 0.71, 95\% CI 0.57 to 0.89 ; $p=0.03$; Fig 4b) and liraglutide (RR 0.41, 95\% CI 0.24 to $0.71 ; p=0.001$ ) when added to metformin.

Hypoglycemia. Compared with placebo, saxaglitpin significantly but slightly increased the incidences of hypoglycemia (RR 1.13, 95\% CI 1.05 to $1.21 ; p=0.0009$; Fig 5a). Compared with sulfonylureas, saxaglitpin significantly reduced the risk of hypoglycemia by $95 \%$ (RR 0.05 , $95 \%$ CI 0.01 to 0.23 ; $p=0.0002$; Fig $5 b$ ). No significant differences were observed in comparison with other active comparators, including other DPP-4 inhibitors.

Body weight. In comparison with placebo, treatment with saxaglipitin was associated with a significant but slight increase of body weight (WMD $0.42 \mathrm{~kg}$, 95\% CI 0.26 to 0.59 ; $p<0.00001$; S4a Fig). Saxagliptin was inferior to liraglutide (WMD $5.10 \mathrm{~kg}, 95 \%$ CI 1.66 to 8.54; $p=0.004$; S4b Fig) and dapagliflozin (WMD $2.40 \mathrm{~kg}$, 95\% CI 1.69 to $3.11 ; p<0.00001$ ). However, treatment with saxagliptin was associated with significantly less effect on body weight than sulfonylureas (WMD $-2.34 \mathrm{~kg}$, $95 \% \mathrm{CI}-3.31$ to -1.36 ; $p<0.00001$ ). In comparison with other DPP-4 inhibitors, changes in body weight were similar.

Other adverse events. The incidences of pancreatitis (RR 1.13, 95\% CI 0.65 to 1.96; $p=0.66$; S5a Fig) and heart failure (RR 0.99, 95\% CI 0.89 to $1.10 ; p=0.85$; S5b Fig) did not differ significantly between saxagliptin and controls (placebo and sulfonylureas). The risk of arthralgia did not differ significantly between saxagliptin and placebo (RR 1.02, 95\% CI 0.92 to $1.13 ; p=0.66$; S5c Fig), Compared with sitagliptin, saxagliptin could significantly reduced the risk of arthralgia (RR $0.20,95 \%$ CI 0.04 to $0.90 ; p=0.04$; S5d Fig), but not compared with other active treatments. Treatment with saxagliptin was not associated with any increased risks of upper respiratory tract infection, urinary tract infection and nasopharyngitis compared with both placebo and active comparators $(p>0.05)$. See S5e-S5j Fig. for forest plots of adverse events above.

\section{Discussion}

This meta-analysis of the available literature on saxagliptin aimed to assess its clinical efficacy and safety in patients with type 2 diabetes. The findings of the analysis indicate that treatment with saxagliptin can lead to significant decreases of HbAlc and FPG compared with placebo, both when given as monotherapy or add-on therapy to other treatments, including metformin, sulfonylureas, thiazolidinediones, dapagliflozin and insulin. Mean placebo-adjusted HbA1c and FPG levels in saxagliptin add-on therapy were lowered by comparable amounts to saxagliptin monotherapy. When combined with submaximal-dose metformin, saxagliptin significantly increased the proportion of patients achieving HbAlc $<7 \%$ compared with acarbose and uptitrated metformin. Generally, efficacy on glycemic control of saxagliptin was similar to sitaglitpin and vildagliptin, while inferior to liraglutide and dapagliflozin. More direct comparisons with other active comparators in future trials may provide further evidence of the efficacy of saxagliptin in comparison with other active treatments.

Saxagliptin is generally well tolerated, with no increased risk of overall and serious adverse events as monotherapy and combination therapy. Additionally, saxagliptin group experienced 
(a)

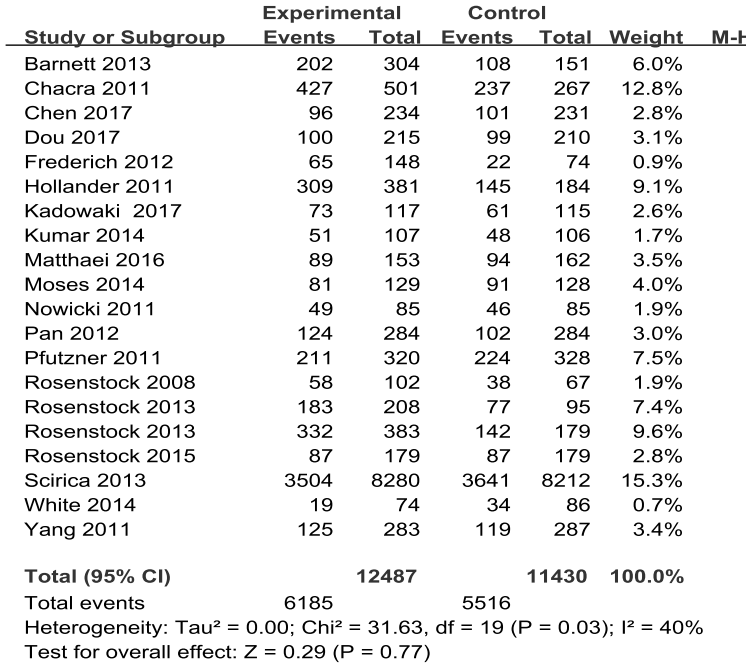

Risk Ratio

Random. $95 \% \mathrm{C}$

$0.93[0.82,1.06]$

$0.96[0.91,1.02]$

$0.94[0.76,1.16]$

$0.99[0.81,1.21]$

$1.48[1.00,2.19]$

$1.03[0.94,1.13]$

$1.18[0.94,1.47]$

$1.18[0.94,1.47]$

$1.00[0.83,1.21]$

$1.00[0.83,1.21]$
$0.88[0.74,1.05]$

$1.07[0.82,1.39]$

$1.22[0.99,1.49]$

$0.97[0.87,1.08]$

$1.00[0.77,1.31]$

$1.09[0.97,1.21]$

$1.09[1.00,1.19]$

$1.00[0.81,1.24]$

$0.95[0.92,0.99]$

$0.65[0.41,1.04]$

$1.07[0.88,1.29]$

$1.01[0.97,1.05]$

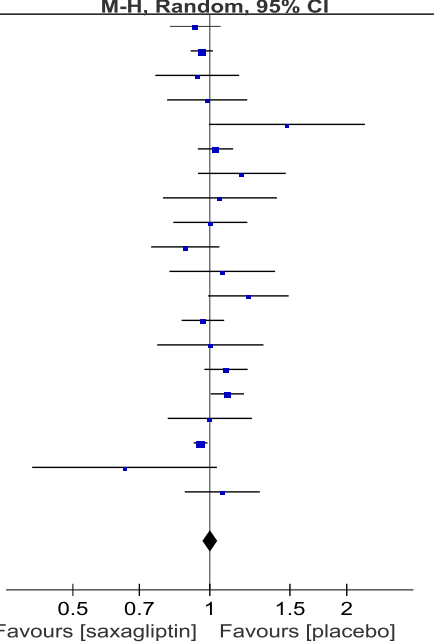

Favours [saxagliptin] Favours [placebo]

(b)

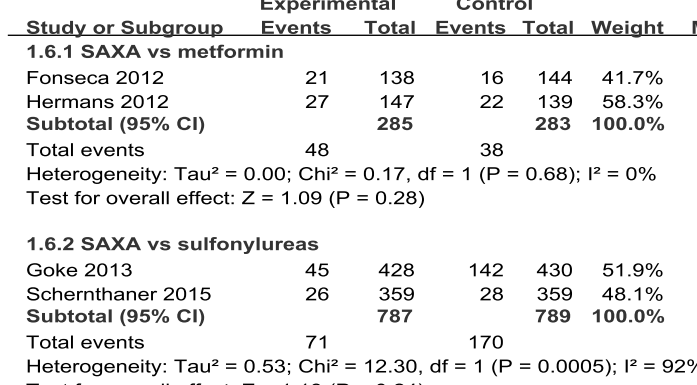

Test for overall effect: $Z=1.18(P=0.24)$

1.6.3 SAXA vs sitagliptin

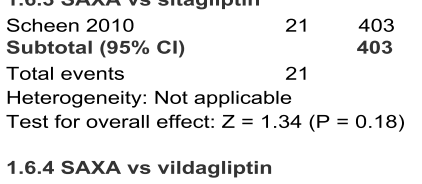

Li $2014 \mathrm{~B}$

Total events 13

Test for overall effect: $Z=0.59(P=0.56)$

1.6.5 SAXA vs acarbose

Du 2017 ( $205 \% \mathrm{Cl})$

Total events

$\begin{array}{lllll}78 & 231 & 109 & 230 & 100.0 \%\end{array}$

$230100.0 \%$

109

$30 \quad 398 \quad 100.0 \%$ $398 \quad 100.0 \%$

Risk Ratio
H, Random, 95\% CI

Risk Ratio

M-H. Random, $95 \%$ CI

$1.37[0.75,2.51]$

$1.16[0.69,1.94]$

$1.24[0.84,1.84]$

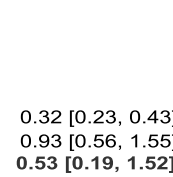

$0.53[0.19,1.52]$

$0.69[0.40,1.19]$ $0.69[0.40,1.19]$

Heterogeneity: Not applicable

Test for overall effect: $Z=2.94(P=0.003)$

1.6.6 SAXA vs dapagliflozin

Rosenstock 2015

Subtotal $(95 \% \mathrm{Cl})$

$93 \quad 176$

176

176
176

93

Heterogeneity: Not applicable

Test for overall effect: $Z=0.80(P=0.42)$

1.6.7 SAXA vs liraglutide

Li 2014 B

Subtotal $(95 \% \mathrm{Cl})$

13

60
60

Total events

13

Test for overall effect: $Z=3.23(P=0.001)$

$0.71[0.57,0.89]$

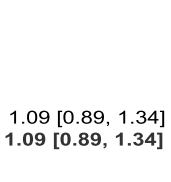

87

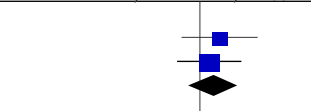

$32 \quad 61 \quad 100.0 \%$

$0.41[0.24,0.71]$ $0.41[0.24,0.71]$

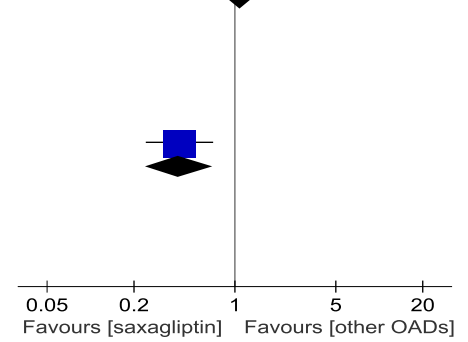

Fig 4. Overall adverse events (a. saxagliptin vs placebo; b. saxagliptin vs active comparators).

https://doi.org/10.1371/journal.pone.0197321.g004 


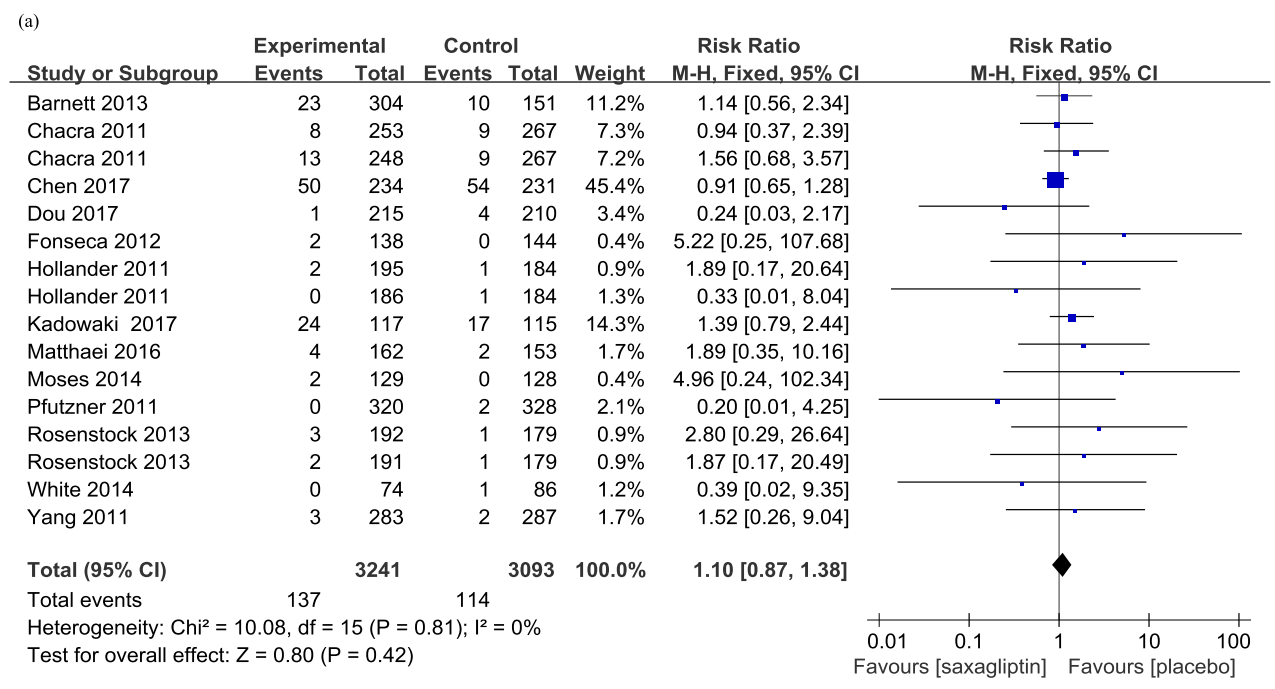

(b)

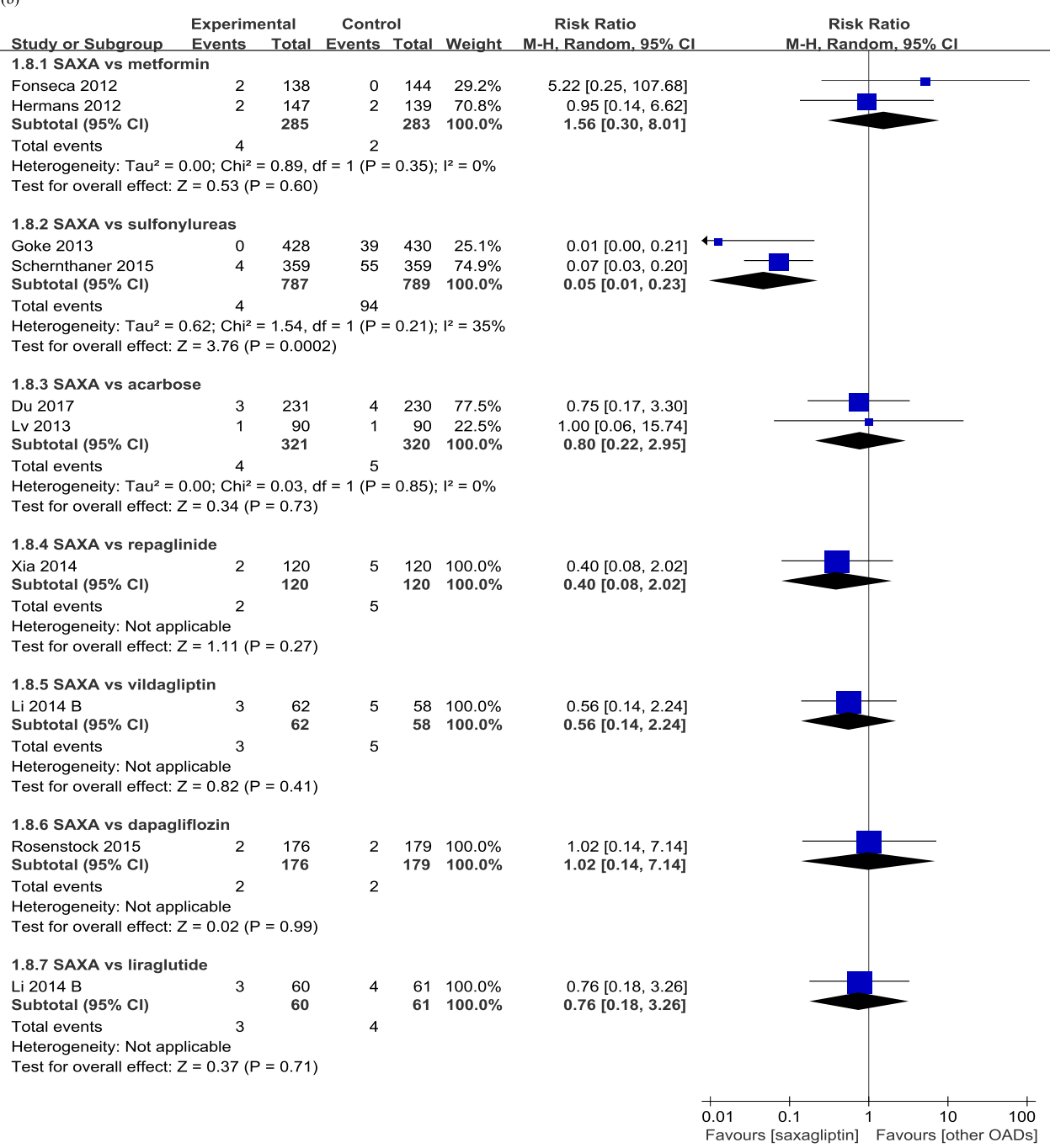

Fig 5. Hypoglycemia (a. saxagliptin vs placebo; b. saxagliptin vs active comparators).

https://doi.org/10.1371/journal.pone.0197321.g005 
less overall adverse events than acarbose and liraglutide groups when combined with metfor$\mathrm{min}$, indicating its favorable safety profile in patients with T2D. Compared with sulfonylureas, saxagliptin had a significant better effect on hypoglycemia and body weight gain. Treatment of saxagliptin was shown to be with a minimal increase of hypoglycemia, which mainly depending on the result from the included Saxagliptin Assessment of Vascular Outcomes Recorded in Patients with Diabetes Mellitus -Thrombolysis in Myocardial Infarction 53 (SAVOR-TIMI 53) trial. Patients in that trial were permitted various concomitant antihyperglycaemic therapies at the doctor's discretion. A post-hoc analysis [52] of SAVOR-TIMI 53 trial found that hypoglycemia rates (any or major) were increased with saxagliptin in patients taking sulfonylureas, not in those taking insulin. A pooled analysis also found that saxagliptin was not associated with increased reported or confirmed hypoglycemia when use of sulfonylurea was excluded [53]. Thus, lower doses of sulfonylureas might be required to reduce risk of hypoglycaemia. Saxagliptin improves the sensitivity of pancreatic islets ( $\alpha$ - and $\beta$-cells) to glucose, inhibits the production of glucagon, and stimulates the secretion of insulin in a glucose-dependent manner. Consequently, it may still be an appropriate agent for patients with relatively higher risk of hypoglycemia.

The potential safety issue of heart failure risk that arose from SAVOR-TIMI 53 and the Examination of Cardiovascular Outcomes with Alogliptin versus Standard of Care (EXAMINE) trials led to the US FDA's recommendation [54] to consider discontinuing saxagliptin and alogliptin for patients if heart failure develops. However, according to our meta-analysis, risk of heart failure was similar with saxagliptin and comparators, with almost identical rates of heart failure in both groups. The result was opposite to previous systematic reviews and meta analyses, among which increased risk of heart failure was found in the treatment of saxagliptin individually or DPP-4 inhibitors as an integrity. Until now, there is no identified pathophysiology for the increased risk of heart failure by saxagliptin treatment. On the contrary, previous preclinical and mechanistic studies of DPP-4 inhibitors suggest additional nonglycemic beneficial actions on blood vessels and the heart, via both GLP-1-dependent and GLP1 -independent effects $[55,56]$. Positive effects of DPP-4 inhibitors on the myocardium have also been described in patients with ischemic heart disease [57].

US FDA had warned that DPP-4 inhibitors may cause serious arthralgia in August 2015, raising safety issues concerning the entire drug class and encouraging healthcare professionals and patients to pay attention [58]. In a search of the FDA Adverse Event Reporting System database, FDA has identified 33 cases of serious arthralgia reported with the use of DPP-4 inhibitors between October 2006 and December 2013. However, the current finding from our meta-analysis showed no safety signal for increased risk of arthralgia in the saxagliptin treatment, and a possible better effect than sitagliptin. Furthermore, there was no increased risk of pancreatitis and infections as supported by our studies, which was also consistent with previous meta-analyses [59-62]. Postmarketing safety surveillance of saxagliptin will provide further data on the incidences of adverse events.

In contrast to GLP-1 receptor agonists, saxagliptin does not mimic infused GLP-1 in its effects on subjective satiety or gastric volume, which have been associated with noticeable weight loss. Overall, saxagliptin appears to be associated with only modest changes in body weight. In comparison with placebo, saxagliptin was associated with a slight gain in body weight, which is of limited clinical significance. However, in comparison with sulfonylureas, saxagliptin had a significant advantage on body weight $(-2.34 \mathrm{~kg})$.

In the US and Europe, saxagliptin has been approved for use in patients with type 2 diabetes, both as monotherapy and in combination with metformin, a sulfonylurea, thiazolidinedione, or insulin, and also in combination with metformin plus insulin. However, in some countries like China, it is currently only approved for use as monotherapy or in combination 
with metformin. Our systematic review has demonstrated significant advantages of saxagliptin in achieving glycemic control when added to sulfonylureas, thiazolidinediones, or insulin, with similar or better safety profiles. These results will help administrators in China and other countries make evidence-based decisions.

This systematic review and meta-analysis of RCTs focused on the efficacy and safety of saxagliptin in the treatment of T2D. The sample size of nearly 30,000 patients enabled the demonstration of reliable results and the comparisons to most classes of oral antidiabetic drugs, by which the knowledge around the comparative efficacy and safety was enriched. However, there are some limitations in this study. Firstly, the follow-up periods of some included trials were relatively short, which limited the observation of longtime outcomes among some comparisons. Secondly, two trials included in the meta-analysis, which comparing saxagliptin with vildagliptin, sitagliptin and (or) liraglutide, were found to have possible high risk of performance and detection bias. This potential bias may reduce the credibility of corresponding results. Interpretations of these findings must be made with caution. More head-to-head trials between saxagliptin and active comparators are still needed to further confirm the efficacy and safety of saxagliptin compared with other classes of antidiabetic drugs.

In conclusion, Generally, saxagliptin has similar efficacy compared with most oral antidiabetic drugs, while may be more effective than acarbose. Saxagliptin is safe in the treatment of T2D, especially having a better safety profile than acarbose and sulfonylureas.

\section{Supporting information}

\section{S1 Text. PRISMA checklist.}

(DOC)

S1 Fig. Risk of bias of included trials (a. graph; b. summary). (EPS)

S2 Fig. Patients achieved HbA1c $<7 \%$ (a. saxagliptin vs placebo; b. saxagliptin vs active comparators).

S3 Fig. Serious adverse events.

S4 Fig. Body weight (a. saxagliptin vs placebo; b. saxagliptin vs active comparators). (EPS)

S5 Fig. Other adverse events [a. pancreatitis; b. heart failure; c. arthralgia (saxagliptin vs placebo); d. arthralgia (saxagliptin vs active comparators); e. upper respiratory tract infection (saxagliptin vs placebo); f. upper respiratory tract infection (saxagliptin vs active comparators); g. urinary tract infection (saxagliptin vs placebo); $h$. urinary tract infection (saxagliptin vs active comparators); i. nasopharyngitis (saxagliptin vs placebo); j. nasopharyngitis (saxagliptin vs active comparators)]. (EPS)

\section{Author Contributions}

Conceptualization: Peng Men, Hui-lin Tang, Suo-di Zhai.

Investigation: Peng Men.

Methodology: Peng Men, Hui-lin Tang. 
Project administration: Suo-di Zhai.

Writing - original draft: Peng Men.

Writing - review \& editing: Xiao-tong Li, Suo-di Zhai.

\section{References}

1. Ogurtsova K, da Rocha Fernandes JD, Huang Y, Linnenkamp U, Guariguata L, Cho NH, et al. IDF Diabetes Atlas: Global estimates for the prevalence of diabetes for 2015 and 2040. Diabetes Res Clin Pract. 2017; 128:40-50. https://doi.org/10.1016/j.diabres.2017.03.024 PMID: 28437734.

2. Zhang $P$, Zhang X, Brown J, Vistisen D, Sicree R, Shaw J, et al. Global healthcare expenditure on diabetes for 2010 and 2030. Diabetes Res Clin Pract. 2010; 87(3):293-301. https://doi.org/10.1016/j. diabres.2010.01.026 PMID: 20171754.

3. Thornberry NA, Gallwitz B. Mechanism of action of inhibitors of dipeptidyl-peptidase-4 (DPP-4). Best Pract Res Clin Endocrinol Metab. 2009; 23(4):479-86. https://doi.org/10.1016/j.beem.2009.03.004 PMID: 19748065.

4. Drucker DJ, Nauck MA. The incretin system: glucagon-like peptide-1 receptor agonists and dipeptidyl peptidase-4 inhibitors in type 2 diabetes. Lancet. 2006; 368(9548):1696-705. https://doi.org/10.1016/ S0140-6736(06)69705-5 PMID: 17098089.

5. Garber AJ, Abrahamson MJ, Barzilay JI, Blonde L, Bloomgarden ZT, Bush MA, et al. Consensus statement by the American Association of Clinical Endocrinologists and American College of Endocrinology on the comprehensive type 2 diabetes management algorithm-2016 executive summary. Endocr Pract. 2016; 22(1):84-113. https://doi.org/10.4158/EP151126.CS PMID: 26731084.

6. Golightly LK, Drayna CC, McDermott MT. Comparative clinical pharmacokinetics of dipeptidyl peptidase-4 inhibitors. Clin Pharmacokinet. 2012; 51(8):501-14. https://doi.org/10.2165/11632930000000000-00000 PMID: 22686547.

7. Li H, Yang L, Tou CK, Patel CG, Zhao J. Pharmacokinetic study of saxagliptin in healthy Chinese subjects. Clin Drug Investig. 2012; 32(7):465-73. https://doi.org/10.2165/11598760-000000000-00000 PMID: 22668067.

8. Li R, Wang R, Li H, Sun S, Zou M, Cheng G. Short-term and long-term effects of dipeptidyl peptidase-4 inhibitors in type 2 diabetes mellitus patients with renal impairment: a meta-analysis of randomized controlled trials. Diabetes Metab Res Rev. 2016; 32(6):460-9. https://doi.org/10.1002/dmrr.2731 PMID: 26433213

9. Kim YG, Min SH, Hahn S, Oh TJ, Park KS, Cho YM. Efficacy and safety of the addition of a dipeptidyl peptidase-4 inhibitor to insulin therapy in patients with type 2 diabetes: A systematic review and metaanalysis. Diabetes Res Clin Pract. 2016; 116:86-95. https://doi.org/10.1016/j.diabres.2016.03.011 PMID: 27321321

10. Foroutan N, Muratov S, Levine M. Safety and efficacy of dipeptidyl peptidase-4 inhibitors vs sulfonylurea in metformin-based combination therapy for type 2 diabetes mellitus: Systematic review and metaanalysis. Clin Invest Med. 2016; 39(2):E48-62. PMID: 27040861.

11. Elgendy IY, Mahmoud AN, Barakat AF, Elgendy AY, Saad M, Abuzaid A, et al. Cardiovascular Safety of Dipeptidyl-Peptidase IV Inhibitors: A Meta-Analysis of Placebo-Controlled Randomized Trials. Am J Cardiovasc Drugs. 2017; 17(2):143-55. https://doi.org/10.1007/s40256-016-0208-x PMID: 27873238.

12. Sjöstrand M, Wei C, Cook W, Johnsson K, Pollack PS, Stahre C, et al. Assessment of Saxagliptin Efficacy: Meta-Analysis of 14 Phase 2 and 3 Clinical Trials. Diabetes Ther. 2017; 8(3):587-99. https://doi. org/10.1007/s13300-017-0261-8 PMID: 28432619.

13. Knobloch $\mathrm{K}$, Yoon U, Vogt $\mathrm{PM}$. Preferred reporting items for systematic reviews and meta-analyses (PRISMA) statement and publication bias. J Craniomaxillofac Surg. 2011; 39(2):91-2. https://doi.org/ 10.1016/j.jcms.2010.11.001 PMID: 21145753.

14. Barnett $A H$, Charbonnel $B$, Donovan $M$, Fleming $D$, Chen $R$. Effect of saxagliptin as add-on therapy in patients with poorly controlled type 2 diabetes on insulin alone or insulin combined with metformin. Curr Med Res Opin. 2012; 28(4):513-23. https://doi.org/10.1185/03007995.2012.665046 PMID: 22313154.

15. Barnett AH, Charbonnel B, Li J, Donovan M, Fleming D, Iqbal N. Saxagliptin add-on therapy to insulin with or without metformin for type 2 diabetes mellitus: 52-week safety and efficacy. Clin Drug Investig. 2013; 33(10):707-17. https://doi.org/10.1007/s40261-013-0107-8 PMID: 23949898.

16. Chacra AR, Tan GH, Apanovitch A, Ravichandran S, List J, Chen R. Saxagliptin added to a submaximal dose of sulphonylurea improves glycaemic control compared with uptitration of sulphonylurea in patients with type 2 diabetes: a randomised controlled trial. Int J Clin Pract. 2009; 63(9):1395-406. https://doi.org/10.1111/j.1742-1241.2009.02143.x PMID: 19614786. 
17. Chacra AR, Tan GH, Ravichandran S, List J, Chen R. Safety and efficacy of saxagliptin in combination with submaximal sulphonylurea versus up-titrated sulphonylurea over 76 weeks. Diab Vasc Dis Res. 2011; 8(2):150-9. https://doi.org/10.1177/1479164111404574 PMID: 21562067.

18. Chen Y, Liu X, Li Q, Ma J, Lv X, Guo L, et al. Saxagliptin add-on therapy in Chinese patients with type 2 diabetes inadequately controlled by insulin with or without metformin: Results from the SUPER study, a randomized, double-blind, placebo-controlled trial. Diabetes Obes Metab. 2018; 20(4):1044-1049. https://doi.org/10.1111/dom.13161 PMID: 29144061.

19. DeFronzo RA, Hissa MN, Garber AJ, Luiz Gross J, Yuyan Duan R, Ravichandran S, et al. The efficacy and safety of saxagliptin when added to metformin therapy in patients with inadequately controlled type 2 diabetes with metformin alone. Diabetes care. 2009; 32(9):1649-55. https://doi.org/10.2337/dc081984 PMID: 19478198.

20. Rosenstock J, Gross JL, Aguilar-Salinas C, Hissa M, Berglind N, Ravichandran S, et al. Long-term 4year safety of saxagliptin in drug-naive and metformin-treated patients with Type 2 diabetes. Diabet Med. 2013; 30(12):1472-6. https://doi.org/10.1111/dme.12267 PMID: 23802840.

21. Dou J, Ma J, Liu J, Wang C, Johnsson E, Yao H, et al. Efficacy and safety of saxagliptin in combination with metformin as initial therapy in Chinese patients with type 2 diabetes: Results from the START study, a multicentre, randomized, double-blind, active-controlled, phase 3 trial. Diabetes Obes Metab. 2018; 20(3):590-598. https://doi.org/10.1111/dom.13117 PMID: 28926170.

22. Du J, Liang L, Fang H, Xu F, Li W, Shen L, et al. Efficacy and safety of saxagliptin compared with acarbose in Chinese patients with type 2 diabetes mellitus uncontrolled on metformin monotherapy: Results of a Phase IV open-label randomized controlled study (the SMART study). Diabetes Obes Metab. 2017; 19(11):1513-20. https://doi.org/10.1111/dom.12942 PMID: 28296055.

23. Fonseca V, Zhu T, Karyekar C, Hirshberg B. Adding saxagliptin to extended-release metformin vs. uptitrating metformin dosage. Diabetes Obes Metab. 2012; 14(4):365-71. https://doi.org/10.1111/j.14631326.2011.01553.x PMID: 22192246.

24. Frederich R, McNeill R, Berglind N, Fleming D, Chen R. The efficacy and safety of the dipeptidyl peptidase-4 inhibitor saxagliptin in treatment-naive patients with type 2 diabetes mellitus: a randomized controlled trial. Diabetol Metab Syndr. 2012; 4(1):36. https://doi.org/10.1186/1758-5996-4-36 PMID: 22828124.

25. Göke B, Gallwitz B, Eriksson J, Hellqvist A, Gause-Nilsson I. Saxagliptin is non-inferior to glipizide in patients with type 2 diabetes mellitus inadequately controlled on metformin alone: a 52-week randomised controlled trial. Int J Clin Pract. 2010; 64(12):1619-31. https://doi.org/10.1111/j.1742-1241.2010. 02510.x PMID: 20846286.

26. Göke B, Gallwitz B, Eriksson JG, Hellqvist A, Gause-Nilsson I. Saxagliptin vs. glipizide as add-on therapy in patients with type 2 diabetes mellitus inadequately controlled on metformin alone: long-term (52week) extension of a 52-week randomised controlled trial. Int J Clin Pract. 2013; 67(4):307-16. PMID: 23638466.

27. Hermans MP, Delibasi T, Farmer I, Lohm L, Maheux P, Piatti P, et al. Effects of saxagliptin added to sub-maximal doses of metformin compared with uptitration of metformin in type 2 diabetes: the PROMPT study. Curr Med Res Opin. 2012; 28(10):1635-45. https://doi.org/10.1185/03007995.2012. 735646 PMID: 23020253.

28. Hollander P, Li J, Allen E, Chen R; CV181-013 Investigators. Saxagliptin added to a thiazolidinedione improves glycemic control in patients with type 2 diabetes and inadequate control on thiazolidinedione alone. J Clin Endocrinol Metab. 2009; 94(12):4810-9. https://doi.org/10.1210/jc.2009-0550 PMID: 19864452.

29. Hollander PL, Li J, Frederich R, Allen E, Chen R; CV181013 Investigators. Safety and efficacy of saxagliptin added to thiazolidinedione over 76 weeks in patients with type 2 diabetes mellitus. Diab Vasc Dis Res. 2011; 8(2):125-35. https://doi.org/10.1177/1479164111404575 PMID: 21562064.

30. Jadzinsky M, Pfutzner A, Paz-Pacheco E, Xu Z, Allen E, Chen R. Saxagliptin given in combination with metformin as initial therapy improves glycaemic control in patients with type 2 diabetes compared with either monotherapy: a randomized controlled trial. Diabetes Obes Metab. 2009; 11(6):611-22. https:// doi.org/10.1111/j.1463-1326.2009.01056.x PMID: 19515181.

31. Pfutzner A, Paz-Pacheco E, Allen E, Frederich R, Chen R; CV181039 Investigators. Initial combination therapy with saxagliptin and metformin provides sustained glycaemic control and is well tolerated for up to 76 weeks. Diabetes Obes Metab. 2011; 13(6):567-76. https://doi.org/10.1111/j.1463-1326.2011. 01385.x PMID: 21342412.

32. Kadowaki T, Muto S, Ouchi Y, Shimazaki R, Seino Y. Efficacy and safety of saxagliptin in combination with insulin in Japanese patients with type 2 diabetes mellitus: a 16-week double-blind randomized controlled trial with a 36-week open-label extension. Expert Opin Pharmacother. 2017 Dec; 18(18):19031919. https://doi.org/10.1080/14656566.2017.1379990 PMID: 28901796. 
33. Kumar KP, Jain SM, Tou C, Schützer K-M. Saxagliptin as initial therapy in treatment-naive Indian adults with type 2 diabetes mellitus inadequately controlled with diet and exercise alone: a randomized, double-blind, placebo-controlled, phase IIlb clinical study. Int J Diab Dev Ctries. 2014; 34(4):201-9.

34. Li CJ, Liu XJ, Bai L, Yu Q, Zhang QM, Yu P, et al. Efficacy and safety of vildagliptin, Saxagliptin or Sitagliptin as add-on therapy in Chinese patients with type 2 diabetes inadequately controlled with dual combination of traditional oral hypoglycemic agents. Diabetol Metab Syndr. 2014; 6:69. https://doi.org/ 10.1186/1758-5996-6-69 PMID: 24917890.

35. Li CJ, Yu Q, Yu P, Zhang QM, Ding M, Liu XJ, et al. Efficacy and safety comparison of add-on therapy with liraglutide, saxagliptin and vildagliptin, all in combination with current conventional oral hypoglycemic agents therapy in poorly controlled Chinese type 2 diabetes. Exp Clin Endocrinol Diabetes. 2014; 122(8):469-76. https://doi.org/10.1055/s-0034-1374586 PMID: 24838155.

36. Lv C, Yu P, Zhou S, Li C, Lv L, Chen R. Efficacy and safety of saxagliptin combined metformin in newly diagnosed type 2 diabetes mellitus patients. Chin J Diab Mel. 2013; 5:759-62.

37. Matthaei S, Catrinoiu D, Celinski A, Ekholm E, Cook W, Hirshberg B, et al. Randomized, Double-Blind Trial of Triple Therapy With Saxagliptin Add-on to Dapagliflozin Plus Metformin in Patients With Type 2 Diabetes. Diabetes care. 2015; 38(11):2018-24. https://doi.org/10.2337/dc15-0811 PMID: 26324329.

38. Matthaei S, Aggarwal N, Garcia-Hernandez P, Iqbal N, Chen H, Johnsson E, et al. One-year efficacy and safety of saxagliptin add-on in patients receiving dapagliflozin and metformin. Diabetes Obes Metab. 2016; 18(11):1128-33. https://doi.org/10.1111/dom.12741 PMID: 27403645.

39. Moses RG, Kalra S, Brook D, Sockler J, Monyak J, Visvanathan J, et al. A randomized controlled trial of the efficacy and safety of saxagliptin as add-on therapy in patients with type 2 diabetes and inadequate glycaemic control on metformin plus a sulphonylurea. Diabetes Obes Metab. 2014; 16(5):443-50. https://doi.org/10.1111/dom.12234 PMID: 24205943.

40. Nowicki M, Rychlik I, Haller H, Warren ML, Suchower L, Gause-Nilsson I. Saxagliptin improves glycaemic control and is well tolerated in patients with type 2 diabetes mellitus and renal impairment. Diabetes Obes Metab. 2011; 13(6):523-32. https://doi.org/10.1111/j.1463-1326.2011.01382.x PMID: 21332627.

41. Nowicki M, Rychlik I, Haller H, Warren M, Suchower L, Gause-Nilsson I, et al. Long-term treatment with the dipeptidyl peptidase-4 inhibitor saxagliptin in patients with type 2 diabetes mellitus and renal impairment: a randomised controlled 52-week efficacy and safety study. Int J Clin Pract. 2011; 65 (12):1230-9. https://doi.org/10.1111/j.1742-1241.2011.02812.x PMID: 21977965.

42. Pan CY, Yang W, Tou C, Gause-Nilsson I, Zhao J. Efficacy and safety of saxagliptin in drug-naive Asian patients with type 2 diabetes mellitus: a randomized controlled trial. Diabetes Metab Res Rev. 2012; 28(3):268-75. https://doi.org/10.1002/dmrr.1306 PMID: 22081481.

43. Rosenstock J, Sankoh S, List JF. Glucose-lowering activity of the dipeptidyl peptidase-4 inhibitor saxagliptin in drug-naive patients with type 2 diabetes. Diabetes Obes Metab. 2008; 10(5):376-86. https:// doi.org/10.1111/j.1463-1326.2008.00876.x PMID: 18355324.

44. Rosenstock J, Aguilar-Salinas C, Klein E, Nepal S, List J, Chen R. Effect of saxagliptin monotherapy in treatment-naive patients with type 2 diabetes. Curr Med Res Opin. 2009; 25(10):2401-11. https://doi. org/10.1185/03007990903178735 PMID: 19650754.

45. Rosenstock J, Hansen L, Zee P, Li Y, Cook W, Hirshberg B, et al. Dual add-on therapy in type 2 diabetes poorly controlled with metformin monotherapy: a randomized double-blind trial of saxagliptin plus dapagliflozin addition versus single addition of saxagliptin or dapagliflozin to metformin. Diabetes care. 2015; 38(3):376-83. https://doi.org/10.2337/dc14-1142 PMID: 25352655.

46. Scheen AJ, Charpentier G, Ostgren CJ, Hellqvist A, Gause-Nilsson I. Efficacy and safety of saxagliptin in combination with metformin compared with sitagliptin in combination with metformin in adult patients with type 2 diabetes mellitus. Diabetes Metab Res Rev. 2010; 26(7):540-9. https://doi.org/10.1002/ dmrr.1114 PMID: 20824678.

47. Schernthaner G, Duran-Garcia S, Hanefeld M, Langslet G, Niskanen L, Ostgren CJ, et al. Efficacy and tolerability of saxagliptin compared with glimepiride in elderly patients with type 2 diabetes: a randomized, controlled study (GENERATION). Diabetes Obes Metab. 2015; 17(7):630-8. https://doi.org/10. 1111/dom.12461 PMID: 25761977.

48. Scirica BM, Bhatt DL, Braunwald E, Steg PG, Davidson J, Hirshberg B, et al. Saxagliptin and cardiovascular outcomes in patients with type 2 diabetes mellitus. N Engl J Med. 2013; 369(14):1317-26. https:// doi.org/10.1056/NEJMoa1307684 PMID: 23992601.

49. White JL, Buchanan P, Li J, Frederich R. A randomized controlled trial of the efficacy and safety of twice-daily saxagliptin plus metformin combination therapy in patients with type 2 diabetes and inadequate glycemic control on metformin monotherapy. BMC Endocr Disord. 2014; 14:17. https://doi.org/ 10.1186/1472-6823-14-17 PMID: 24565221.

50. Xia C, Peng H, Zhang G. Clinical trial of saxagliptin combined metformin in newly diagnosed type 2 diabetes mellitus patients. Chinese Remedies \& Clinics. 2014; 14(5):640-2. 
51. Yang W, Pan CY, Tou C, Zhao J, Gause-Nilsson I. Efficacy and safety of saxagliptin added to metformin in Asian people with type 2 diabetes mellitus: a randomized controlled trial. Diabetes Res Clin Pract. 2011; 94(2):217-24. https://doi.org/10.1016/j.diabres.2011.07.035 PMID: 21871686.

52. Cahn A, Raz I, Mosenzon O, Leibowitz G, Yanuv I, Rozenberg A, et al. Predisposing Factors for Any and Major Hypoglycemia With Saxagliptin Versus Placebo and Overall: Analysis From the SAVORTIMI 53 Trial. Diabetes care. 2016; 39(8):1329-37. https://doi.org/10.2337/dc15-2763 PMID: 27222508.

53. Hirshberg B, Parker A, Edelberg H, Donovan M, lqbal N. Safety of saxagliptin: events of special interest in 9156 patients with type 2 diabetes mellitus. Diabetes Metab Res Rev. 2014; 30(7):556-69. https:// doi.org/10.1002/dmrr.2502 PMID: 24376173.

54. US food and drug administration. Safety Alerts for Human Medical Products: Diabetes Medications Containing Saxagliptin and Alogliptin: Drug Safety Communication—Risk of Heart Failure. 2015 [cited 2016 Aug 17]. https://www.fda.gov/Safety/MedWatch/Safetylnformation/ SafetyAlertsforHumanMedicalProducts/ucm494252

55. Scheen AJ. Cardiovascular effects of dipeptidyl peptidase-4 inhibitors: from risk factors to clinical outcomes. Postgrad Med. 2013; 125(3):7-20. https://doi.org/10.3810/pgm.2013.05.2659 PMID: 23748503.

56. Jose T, Inzucchi SE. Cardiovascular effects of the DPP-4 inhibitors. Diab Vasc Dis Res. 2012; 9 (2):109-16. https://doi.org/10.1177/1479164111436236 PMID: 22337893

57. Karagiannis T, Paschos $P$, Paletas K, Matthews DR, Tsapas A. Dipeptidyl peptidase-4 inhibitors for treatment of type 2 diabetes mellitus in the clinical setting: systematic review and meta-analysis. BMJ. 2012; 344:e1369. https://doi.org/10.1136/bmj.e1369 PMID: 22411919.

58. US Food and Drug Administration. FDA drug safety communication: FDA warns that DPP-4 inhibitors for type 2 diabetes may cause severe joint pain. 2015 [cited 2016 Aug 17]. http://www.fda.gov/Drugs/ DrugSafety/ucm459579

59. Wang T, Wang F, Gou Z, Tang H, Li C, Shi L, et al. Using real-world data to evaluate the association of incretin-based therapies with risk of acute pancreatitis: a meta-analysis of 1,324,515 patients from observational studies. Diabetes Obes Metab. 2015; 17(1):32-41. https://doi.org/10.1111/dom.12386 PMID: 25200423.

60. Monami M, Dicembrini I, Mannucci E. Dipeptidyl peptidase-4 inhibitors and pancreatitis risk: a metaanalysis of randomized clinical trials. Diabetes Obes Metab. 2014; 16(1):48-56. https://doi.org/10.1111/ dom.12176 PMID: 23837679.

61. Giorda CB, Sacerdote C, Nada E, Marafetti L, Baldi I, Gnavi R. Incretin-based therapies and acute pancreatitis risk: a systematic review and meta-analysis of observational studies. Endocrine. 2015; 48 (2):461-71. https://doi.org/10.1007/s12020-014-0386-8 PMID: 25146552.

62. Yang W, Cai X, Han X, Ji L. DPP-4 inhibitors and risk of infections: a meta-analysis of randomized controlled trials. Diabetes Metab Res Rev.2016; 32(4):391-404. https://doi.org/10.1002/dmrr.2723 PMID: 26417956. 